\title{
LA METACOGNICIÓN Y EL MEJORAMIENTO DE LA ENSEÑANZA DE QUÍMICA UNIVERSITARIA
}

\author{
Par ol o, M.E. ${ }^{1}$; Bar bie r i, L.M. ${ }^{1}$ y Ch r obak, R. ${ }^{2}$ \\ ${ }^{1}$ Escuela Superior de Salud y Ambiente \\ ${ }^{2}$ Facultad de Ingeniería \\ Universidad Nacional del Comahue. Buenos Aires 1400 (8300) Neuquén. Argentina \\ lbradach@neunet.com.ar
}

\begin{abstract}
Resumen. En este trabajo, que es parte de una investigación más extensa, sobre mejoramiento de la enseñanza de química universitaria, se presentan algunos resultados obtenidos luego de aplicar una nueva propuesta de enseñanza, destinada a la comprensión y resolución de problemas sobre el tema «Soluciones». Con el objeto de facilitar el aprendizaje significativo, la propuesta de trabajo incluye el uso de las denominadas herramientas metacognitivas que permitan aplicar metodologías conducentes al logro de dichos aprendizajes por parte de los estudiantes.

Luego de aplicar las mencionadas herramientas, se procedió a realizar la evaluación de los estudiantes participantes para obtener datos sobre los logros alcanzados y sus aprendizajes. El análisis de los resultados muestra que el uso del nuevo enfoque instruccional ayuda a los estudiantes en sus procesos de aprendizaje, en la medida que se vayan haciendo conscientes de los mecanismos que se utilizan para obtener aprendizaje significativo.
\end{abstract}

Palabras clave. Metacognición, herramientas metacognitivas, soluciones, aprendizaje significativo, enseñanza universitaria.

Summary. This work, which is part of a more extensive research project on the improvement of Chemistry teaching at university level, presents the results obtained by applying an innovative teaching methodology. This methodology was designed with the objective of helping students to better understand and solve problems regarding the topic «Solutions». In order to facilitate learning, the proposed methodology includes the use of metacognitive tools (concept maps, Gowin's Vee and clinical interviews), which allows the students to apply significant learning methodologies.

After applying these tools, we evaluated the students in order to measure their achievements and their learning. The analysis of the results shows that the use of this new instructional approach helps the students in their learning process because they become aware of the mechanism they use to achieve significant learning.

K eywords. Metacognition, metacognitive tools, solutions, significant learning, university teaching.

\section{ALGUNOS ANTECEDENTES}

Durante los tiempos que corren, es incuestionable la marcada influencia que ejercen las ideas y los procedimientos de las ciencias exactas y naturales en la vida cotidiana de las personas. Por este motivo, gran parte de las carreras universitarias, cuyo tema central no reside en las ciencias exactas y naturales, incluyen al menos uno o dos cursos de estas materias, como, por ejemplo, la química. Resulta obvia, entonces, la importancia que reviste la comprensión de conceptos químicos por parte de los estudiantes, no sólo de química, sino también de otras especialidades. Sin embargo, este objetivo se logra sólo en parte o, al menos, no se logra en la medida en que sería conveniente. Por ello más que dar información, la educación debe formar al estudiante para que sea capaz de formular preguntas y encontrar respuestas. Es así que en la actualidad, las técnicas de enseñanza no parecen cubrir las necesidades de los estudiantes. Es nuestro criterio que la razón principal de estas deficiencias consiste en que pocas veces los estudiantes (y aun muchos profesores) son conscientes de la importancia que tiene para ellos reflexionar sobre sus propios saberes y sobre la forma en que se producen los conocimientos, acción que ha dado en llamarse metacognición. 
Por lo tanto, estimamos que el desafío actual para las universidades consiste en proveer experiencias que mantengan el interés del estudiante y les faciliten el desarrollo de habilidades de orden superior. Creemos que una forma de lograr este objetivo es a través de la aplicación de modelos de instrucción que motiven a los educandos para la construcción de los conceptos centrales del tema en estudio.

Por lo expuesto y debido a las numerosas dificultades que presenta la enseñanza de química, urge, tanto a los profesores como a los estudiantes, lograr una mayor efectividad de los modelos de enseñanza-aprendizaje que se utilizan en la actualidad. A esta urgencia intenta responder el presente trabajo, adoptando un modelo de instrucción (Chrobak y Herrera, 1996) apoyado por fundamentos teóricos sobre el aprendizaje de ciencias y en experiencias de clase, realizadas con estudiantes de grado en los cursos normales que se dictan en la Universidad Nacional del Comahue.

\section{LA TEORÍA AUSUBELIANA COMO ORIGEN DE LAS HERRAMIENTAS METACOGNITIVAS}

Las ideas básicas en que se fundamenta el presente trabajo fueron presentadas por primera vez por Ausubel en su libro Educational Psychology, a Cognitive View (1978). Describiremos muy brevemente las más importantes conclusiones que surgen de las ideas y principios ausubelianos y algunas de sus aplicaciones para el mejoramiento de los modelos de instrucción. Ausubel colocó el énfasis en la idea del aprendizaje significativo y sostiene que, para que éste ocurra, el alumno debe ser consciente de que él debe relacionar las nuevas ideas o informaciones que quiere aprender, a los aspectos relevantes y pertinentes de su estructura cognoscitiva (principio de la inclusión). Esto debe realizarse en forma no arbitraria, no al pie de la letra, sino substancialmente. Para que ello sea posible se deben cumplir ciertos requisitos, siendo los más importantes: $a$ ) el material a ser aprendido debe ser potencialmente significativo, esto quiere decir que, por ejemplo, no se pueden enseñar significativamente listas de palabras sin sentido, las cuales, al no tener significado inherente, no podrán ser relacionadas con otros conceptos ya existentes (conocimiento previo) en la estructura cognoscitiva; $b$ ) el que está aprendiendo debe poseer conceptos y proposiciones relevantes en su estructura cognoscitiva, que sean capaces de actuar como «base de anclaje» para las nuevas ideas a ser asimiladas; $c$ ) el que está aprendiendo debe elegir relacionar intencionadamente el material en potencia significativo en forma no arbitraria y substantiva con la estructura cognoscitiva que él ya posee. Si alguna de estas condiciones falla, el aprendizaje significativo también se verá afectado.

Dado que el modelo de instrucción aplicado incluye la utilización de mapas conceptuales, la u ve de Gowin y las entrevistas clínicas, se hará una breve revisión de los antecedentes existentes respecto de estos instrumentos y su efectividad para lograr aprendizajes significativos.

Una excelente y más completa explicación de los orígenes y alcances de los mapas conceptuales, de la u ve de Gowin y de las entrevistas clínicas, podemos encontrarla, entre muchos otros, en los trabajos Aprendiendo a aprender, de Novak y Gowin (1988), en «Instrumento de pesquisa em ensino y aprendizaje», de Moreira y Da Silveira (1993), y en «Uso de estrategias facilitadoras del aprendizaje significativo en los cursos de física introductoria», de Chrobak (1995).

\section{LOS MAPAS CONCEPTUALES}

El mapa conceptual es un recurso gráfico esquemático que, por su forma constitutiva, sirve para representar un conjunto de significados conceptuales incluidos en una estructura de proposiciones. Con ellos se pueden distinguir los procesos de organización jerárquica, la diferenciación progresiva y la reconciliación integradora, que son los principios básicos del aprendizaje significativo. Este hecho de que con los mapas conceptuales es posible la «visualización gráfica» del cumplimiento y control (autorregulación) de estos principios es el principal motivo para que se constituyan en una herramienta insustituible cuando se trata de ayudar a los estudiantes a aprender a aprender.

Los estudiantes pueden aprender conceptos poco familiares memorizándolos. Una definición, por ejemplo, puede ser aprendida repitiéndola una y otra vez hasta ser capaz de poner las palabras correctas en el orden apropiado. Se puede elegir, en cambio, integrar la nueva información con lo que ya se sabe. Elaborar mapas conceptuales facilita un aprendizaje lleno de significado, ya que requiere que se realicen importantes decisiones acerca de: $a$ ) la importancia de las ideas; $b$ ) cómo estas ideas se relacionan unas con otras; y $c$ ) cómo estas ideas se relacionan con los conocimientos previos. Además se sabe que la mente humana organiza los conceptos en forma jerárquica, por lo que el mapa debe ser organizado también de esta forma, lo que requiere el esfuerzo de distinguir la jerarquía u orden de importancia de los conceptos, para así distribuirlos en el mapa.

En resumen, podemos decir que la construcción de un mapa conceptual requiere, del que aprende, seguir los siguientes pasos:

1) Identificar los conceptos centrales (o relevantes).

2) Establecer un orden jerárquico para los mismos.

3) Relacionar los conceptos entre sí por medio de palabras de enlace o nexos, a fin de formar proposiciones, que son las que otorgan significado a los conceptos.

4) Buscar, dentro de la estructura formada, enlaces transversales.

5) Examinar la estructura final del mapa realizando los ajustes necesarios.

Cuando se aplican los mapas conceptuales como herramienta, se logra un aprendizaje más activo, porque el que 
aprende autorregula conscientemente la construcción de los nuevos conceptos a partir de sus conocimientos anteriores.

Es por eso que podemos afirmar que los mapas conceptuales son facilitadores de un aprendizaje significativo, se basan en las relaciones entre ideas y permiten usar lo que ya se sabe mientras se aprende una idea nueva (Novak, 1996; Moreira, 1993, 1998a).

\section{LA UVE DE GOWIN}

Así como los mapas conceptuales permiten abordar los aspectos relacionados con la estructura de conocimientos, Gowin desarrolló la uve, que se puede utilizar como estrategia de análisis, tanto de la construcción (conocimientos científicos) como de la reconstrucción (aprendizaje) de los mismos. En efecto, esta técnica heurística fue desarrollada para ayudar a profesores y estudiantes a comprender la naturaleza y producción de conocimientos y consta básicamente de doce elementos «epistémicos» cuya explicitación permite a los seres humanos clarificar y reflexionar sobre sus propios procesos de aprendizaje.

Un gran número de filósofos de la ciencia (Kuhn, 1962) ha aceptado el punto de vista de que toda observación está sesgada por la teoría. Este punto de vista ha servido para negar el enfoque de los primeros positivistas lógicos que afirmaban que las teorías deben abandonarse cuando no están apoyadas por los hechos. Sin embargo, la opinión de que las observaciones están cargadas de significado teórico están teniendo cada vez más aceptación, aunque todavía es, en parte, confusa. El diagrama u ve y sus definiciones de concepto y hecho nos muestran cómo evitar esta confusión, ya que son elementos diferentes en la estructura de conocimiento. En cualquier nuevo caso de construcción de conocimiento se deben integrar los conceptos con las regularidades en los hechos. A través de los registros podemos construir hechos que sean repetibles, fiables y consistentes, pero que en sí no tienen significado, ya que no hay nada en el aspecto externo de los acontecimientos que nos diga qué significan; por ello es necesario tener ideas, teorías, estructuras conceptuales, visiones del mundo, etc. para tener éxito en esta tarea de la comprensión humana.

Los diagramas uve representan el intento que puede hacer el estudiante para expresar cómo se construye algún fragmento de conocimiento que, a su vez, permite englobar ciertos principios epistemológicos. A medida que se practica con la u ve, se enriquece notablemente el significado de las lecturas filosóficas que tratan de la naturaleza del conocimiento y, en forma recíproca, algunas de estas lecturas nos ayudarán a comprender e interpretar las respuestas de los estudiantes y obtener una mayor comprensión de los elementos de la uve. Esto incrementa la seguridad acerca de cómo y por qué es valioso el uso de una técnica de este tipo. Muchos estudiantes llegan a interesarse en actividades como la epistemología a partir de este tipo de enseñanza (Novak, 1998a, 1998b; Moreira, 1993).

\section{LA ENTREVISTA CLÍNICA}

Uno de los primeros escollos que debe sortear el docente que intenta avanzar hacia un aprendizaje significativo es determinar lo que el alumno ya conoce, es decir, sus conocimientos previos (donde estarán incluidas evidentemente las concepciones alternativas) y, para ello, las pruebas tradicionales de diagnóstico se revelaron demasiado rígidas e insuficientes. Fue menester encontrar otro instrumento que permitiera averiguar los conocimientos previos de los alumnos con certeza, ductilidad y economía. Se deben determinar cuáles son los conceptos más importantes (centrales) relacionados con el material a enseñar que se encuentran firmemente establecidos en la memoria de largo plazo, así como también la forma en que están relacionados entre ellos. Estos conceptos tienen la particularidad de ser claros y estables para el estudiante, siendo, por lo tanto, los más pertinentes (en términos de la teoría de la asimilación) para actuar como subsumsores del nuevo material o, lo que es lo mismo, para funcionar como elementos de «anclaje» donde la nueva información que se va a impartir quedará firmemente «encadenada».

Para el logro de tales fines, el profesor Novak, de la Universidad de Cornell, rescató la entrevista clínica, utilizada originalmente por Piaget, la adaptó con esta nueva finalidad y la utilizó ampliamente, tanto para determinar el conocimiento previo como para verificar los avances de los estudiantes a lo largo del proceso de instrucción. Entendemos, como tal, el desarrollo o crecimiento de la estructura cognitiva de los estudiantes; es decir, lo que realmente debe ser el foco de máximo interés de la educación y del docente preocupado por sus estudiantes: el logro de aprendizajes significativos. La evidencia de que este tipo de aprendizaje sea el que realmente está ocurriendo, sólo se tendrá conociendo la evolución de la estructura cognitiva del alumno en el área de conocimientos que se está impartiendo. De esta forma podemos decir que, al menos hasta el presente, no se conoce mejor alternativa, para evaluar el desarrollo de las estructuras conceptuales de los estudiantes, que la combinación de las técnicas de entrevista clínica con la elaboración de los correspondientes «mapas cognitivos», entendiendo, por tales, los mapas conceptuales elaborados por los docentes a partir de las respuestas de los estudiantes obtenidas mediante las entrevistas. Es necesario aclarar que, en rigor, los mapas que resultan no son ciento por ciento cognitivos, ya que, para que lo sean, deberían ser confeccionados por los propios estudiantes. No obstante, con buena práctica de parte del docente, resultan una herramienta de alta utilidad cuando lo que realmente se persigue es atender el seguimiento del aprendizaje.

En la actualidad está ampliamente aceptado que la entrevista clínica es un excelente instrumento de investigación educativa, primordialmente en la enseñanza de ciencias exactas y naturales, que se ha revelado irreemplazable para la determinación de estructuras cognitivas como así también para la detección de las concepciones alternativas de los estudiantes (Novak y Gowin, 1986). 


\section{DESCRIPCIÓN DE LA INVESTIGACIÓN}

Con el objeto de mejorar la calidad de la enseñanza universitaria, un grupo de docentes de la Universidad Nacional del Comahue (UNCo) estamos trabajando en la aplicación de herramientas metacognitivas que se desarrollan a partir del marco teórico esbozado en las secciones anteriores, como son los mapas conceptuales, las entrevistas clínicas y la u ve heurística de Gowin. Dichas herramientas se utilizan en la planificación, seguimiento y evaluación del proceso de enseñanza-aprendizaje, ya que son ampliamente reconocidas como estrategias que facilitan el aprendizaje significativo al permitir indagar acerca de nuestro propio conocimiento.

El objetivo que se persigue es que el alumno descubra por sí mismo la utilidad de las estrategias y desarrolle su metacognición, conociendo qué formas de actuación mental son más eficaces en cada situación; es decir, que aprenda a aprender. El alumno que ha aprendido a aprender sabe trabajar por sí mismo, descubre nuevas técnicas y autorregula su sistema de trabajo sin la guía constante de otra persona.

Si lo que se desea es sólo que los alumnos aprendan, la instrucción mecánica puede ser suficiente; si se aspira a que aprendan a aprender, el método didáctico debe ser metacognitivo. Otro aspecto importante que no deben olvidar los profesores consiste en hacerles ver a los alumnos la ventaja de formularse preguntas sobre los temas centrales de la asignatura. Esta misma reflexión es aplicable a cualquier otra técnica o recurso de aprendizaje.

Se trabajó con alumnos del primer año de la carrera de licenciatura en Saneamiento y Protección Ambiental que cursaban la asignatura Química I. La unidad elegida fue «Soluciones», ya que los conceptos adquiridos durante el desarrollo de la misma son inclusores de nuevos conceptos y permitirían a los alumnos aplicarlos a la resolución de situaciones problemáticas en la vida cotidiana y profesional futura.
En todo momento, a través de la metodología de trabajo empleada se promovió la participación activa del alumno, guiado por el docente, en la construcción de su propia estructura cognitiva influenciada por el contexto social y temporal en que se desarrolló la experiencia.

Sobre las bases del aprendizaje significativo de Ausubel, Novak y Gowin, construimos un mapa conceptual para ordenar y relacionar los contenidos de la unidad (Anexo I), una u ve heurística que nos permitió organizar el presente trabajo (A) y una u ve que contempla el desarrollo del tema «Soluciones» en clases teóricas y prácticas (B) (Fig. 1).

\section{UVE HEURÍSTICA UTILIZADA PARA ORGANI- ZAR ESTA INVESTIGACIÓN EDUCATIVA}

1) Pregunta central. ¿Cómo se enriqueció la estructura cognitiva de los alumnos sobre el tema «Soluciones» luego de haber desarrollado la unidad en clase con la utilización de herramientas metacognitivas?

2) Objeto/evento. Entrevistas y mapas conceptuales realizadas por los alumnos de Química I de la licenciatura en Saneamiento y Protección Ambiental de la Escuela Superior de Salud y Ambiente (ESSA), de la UNCo.

3) Cosmovisión. El conocimiento de la propia estructura cognitiva ayuda al alumno a la incorporación de nuevos conceptos y a la relación de los mismos con el fin de aplicarlos a la resolución de situaciones problemáticas en la vida cotidiana y profesional futura.

4) Filosofía. El aprendizaje es un proceso dinámico que implica una participación activa del alumno guiado por el docente.

El conocimiento se va construyendo sobre una estructura preexistente que aporta cada individuo y que está influenciada por el contexto social y temporal en el que se encuentra.

\begin{tabular}{|llll|}
\hline CONCEPTUAL & METODOLÓGICO \\
\hline 3 Cosmovisión & Pregunta central & Afirmaciones de valor \\
5 & Teoría & 10 Afirmaciones \\
de conocimiento
\end{tabular}


5) Teoría. Ausubel, Novak, Gowin.

6) Principios:

- El aprendizaje significativo se logra cuando hay una predisposición a aprender, cuando se trabaja con materiales significativos y cuando el conocimiento a impartir es relevante.

- El conocimiento previo influencia todo nuevo aprendizaje.

- Es importante conocer las concepciones alternativas a fin de corregirlas o ampliarlas.

- El contexto tiene fuerte influencia sobre el aprendizaje y su transferencia.

- El responsable de construir su estructura cognitiva es cada individuo; esto le permitirá entender el mundo que lo rodea y participar activamente del mismo.

7) Conceptos. Aprendizaje significativo, mapas conceptuales, entrevistas clínicas, u ve heurística, estructura cognitiva, construcción social, conocimiento previo, constructivismo.

8) Registros. La cátedra elaboró una guía para la construcción de mapas conceptuales que fue leída y analizada durante el transcurso de la clase.

Se invitó a trabajar en forma individual o grupal en la construcción de un mapa conceptual sobre una lista elaborada con conceptos previos. Se realizaron 33 mapas, algunos de ellos fueron entregados con el nombre de los autores y otros en forma anónima.

Se trató el tema «Soluciones» en clases teóricas y prácticas (Laboratorio y resolución de problemas). Al término de la unidad se solicitó a los alumnos que trataran de enriquecer el mapa construido inicialmente, en forma grupal, con todos los conceptos discutidos durante el desarrollo del tema.

Se elaboró una guía de preguntas para una entrevista clínica, cuya primera parte consignaba los datos personales de los encuestados y luego la parte específica consistía en seis preguntas sobre el tema «Soluciones».

Se invitó a realizar una entrevista clínica a 11 alumnos cuyo rendimiento en el primer examen parcial había sido heterogéneo. Las entrevistas fueron grabadas y cada una tuvo una duración aproximada de diez minutos. Los alumnos provenían de establecimientos educativos con distintas orientaciones (escuelas comerciales, técnicas y bachilleratos). A modo de ejemplo, se presentan los anexos II, III y IV, que corresponden al proceso de aprendizaje de una de las alumnas entrevistadas.

9) Transformaciones. A partir de una lista de los conceptos expresados por el entrevistado, construimos un mapa conceptual de cada entrevista (Anexo IV).
Dentro del grupo de alumnos entrevistados, el 50\% evidenció dificultades de expresión oral y en el $60 \%$ de los casos se observó la persistencia de concepciones alternativas.

\section{0) Afirmaciones de conocimiento.}

\section{Análisis del proceso de aprendizaje de una alumna entrevistada}

En el mapa conceptual individual realizado por una alumna entrevistada previo al tratamiento del tema en clase (Anexo II), se observaron dificultades en la relación de conceptos, debido a la falta de experiencia en el uso de esta herramienta metacognitiva. En el aspecto conceptual, existió una errónea vinculación entre el concepto de concentración y los de solución saturada, diluida o sobresaturada y entre el concepto de concentración y la capacidad de conducir la electricidad, lo que evidenció la existencia de una concepción alternativa, ya que no todas las soluciones tienen esta propiedad.

La alumna intervino en la construcción de un mapa grupal donde se observó que se relacionaban los conceptos en forma más completa. La relación establecida en el mapa individual entre concentración y los conceptos de solución saturada, diluida o sobresaturada persistió, lo cual podría deberse a un problema de mala elección del conector. Corrigió la concepción alternativa que relacionaba concentración con capacidad de conducir la electricidad, logrando una mejor organización de los conceptos y del uso de los conectores.

Nos resultó complejo el armado del mapa conceptual construido con las respuestas de la alumna en la entrevista, ya que fue muy rico en relaciones conceptuales.

Observamos las siguientes concepciones alternativas:

- La solubilidad es máxima cuando se considera que el soluto y el solvente tienen el mismo estado de agregación.

- Las soluciones son mezclas de líquidos miscibles (excluye otros tipos de soluciones).

- Considerar la presencia de un catalizador como un factor que afecta a la solubilidad.

A lo largo del cursado de la materia, la alumna se mostró interesada y participativa.

\section{Análisis general de los procesos de aprendizaje de los alumnos entrevistados}

Al hacer un análisis general en que se consideraron los procesos de aprendizaje de todos los alumnos entrevistados, observamos que, en los mapas conceptuales realizados antes del tratamiento del tema en clase, se evidenció falta de experiencia en el uso de esta herramienta metacognitiva, debido a la ausencia de nexos, mala asignación de jerarquía conceptual y dificultades en la elección 
de conceptos centrales a partir de una frase conceptual. Era la primera vez que gran parte de los alumnos aplicaba esta técnica de trabajo.

Al comparar los mapas realizados al comienzo y al finalizar la unidad, se observó que los mapas conceptuales grupales, posteriores al desarrollo del tema, mostraron mayor cantidad de conceptos y presentaron una mayor riqueza en relaciones, lo que hizo más clara su interpretación.

Si bien notamos un mejor uso de la herramienta, la escasa experiencia demostró la persistencia de dificultades en relacionar conceptos, en establecer un orden jerárquico y de concepciones alternativas.

Las entrevistas también evidenciaron un enriquecimiento de la estructura cognitiva si bien algunas concepciones alternativas persistieron. Dada las características de la técnica, algunos de los alumnos mostraron nerviosismo y dificultades en la expresión oral.

11) Afirmaciones de valor. La u ve heurística nos resultó útil para planificar el trabajo presente. Creemos que puede ser aplicable a la planificación y para la evaluación de un proceso educativo.

El mapa conceptual es una herramienta práctica porque representa gráficamente una estructura conceptual que nos permite evidenciar en el alumno conceptos previos, concepciones alternativas, qué relaciones establece entre conceptos y también evidenciar problemas en la construcción del mapa (jerarquía respetada, elección del concepto central, formar frases con sentido, etc.). En la práctica docente nos permite planificar desde una unidad hasta un programa completo de la materia y hacer una evaluación periódica del proceso de aprendizaje durante el cuatrimestre. Para los alumnos puede resultar una herramienta útil al ser aplicado al estudio de un tema particular, para relacionar conceptos de distintas unidades, para enriquecer y cambiar concepciones alternativas.

La mayor riqueza en relaciones observada en los mapas realizados en forma grupal, creemos que se debe no sólo al desarrollo del tema en clase sino a la construcción social del conocimiento que se logró trabajando de esta forma.

Los mapas conceptuales construidos a partir de las entrevistas clínicas, no podemos asegurar que reflejen la estructura cognitiva de los alumnos, ya que en la construcción del mapa no podemos evitar ser subjetivos. Otras veces la duda se establece en si el alumno tiene una concepción alternativa o dificultades en la expresión oral del concepto, además del hecho de sentirse evaluados al responder la entrevista clínica, a pesar de habérseles aclarado que no era una instancia evaluativa.

Si bien el análisis de las entrevistas insume mucho tiempo, la riqueza de la información que se obtiene lo justifica.
Para el alumno, construir un mapa conceptual y ser entrevistado por el docente constituyen situaciones en las que tiene que expresar, en forma escrita u oral, los conceptos aprendidos de manera clara y ordenada. Esto lo ayudará en el futuro en sus procesos de aprendizaje a medida que se haga consciente de los mecanismos que utiliza para tal fin.

Pudimos apreciar que, instando a los alumnos a verbalizar sus conocimientos y en particular los conceptos centrales adquiridos en forma clara y estable, se favorece la reflexión metacognitiva y se aprovecha el conocido hecho que establece que un conocimiento fue adquirido signitivamente cuando se puede expresar con palabras sencillas y que pertenecen al bagaje de vocabulario del alumno; o sea, expresarlo con sus propias palabras. Cuando esto se logra, se adquieren niveles de conceptualización más elevados que van más allá de la simple redacción escrita o de realizar gráficos que muestren relaciones entre dos variables (Feyman, 1959).

\section{UVE HEURÍSTICA QUE CONTEMPLA EL DE- SARROLLO DEL TEMA «SOLUCIONES»}

1) Pregunta central. ¿Qué son las soluciones?

2) Objeto/evento. Desarrollo de una unidad de la asignatura Química I en la carrera de licenciatura en Saneamiento y Protección Ambiental.

3) Cosmovisión: El tema «Soluciones» resulta crucial para el desarrollo de la asignatura Química I.

4) Filosofía. Una fuerte formación en ciencias básicas y en particular de Química I es de fundamental importancia para estudiar los problemas del saneamiento ambiental.

5) Teoría. Teoría de las soluciones (Chang, 1997; Whitten, 1999; Cárdenas, 1998).

\section{6) Principios:}

- Las soluciones son sistemas homogéneos formados por uno o más solutos en un solvente.

- La concentración de una solución expresa la cantidad de soluto disuelta en una determinada cantidad de solvente o solución.

- Facilitando la interacción soluto-solvente se favorece la solubilidad.

7) Conceptos. Soluciones, concentración, electrolito, soluto, solvente, solubilidad, solución diluida, solución saturada, solución sobresaturada, sistemas homogéneos.

8) Registros. Guías para la elaboración de mapas conceptuales. Mapas conceptuales realizados por los alumnos como herramienta de estudio. Apuntes de clases teóricas. Trabajos prácticos de laboratorio y elaboración de sus correspondientes informes. Resolución de problemas. 
9) Transformaciones. Resultados de las evaluaciones escritas correspondientes a exámenes parciales y finales. Resultados de los cuestionarios de trabajos prácticos.

10) Afirmaciones de conocimiento. Conceptos manifestados por los alumnos luego del desarrollo del tema en clase:

- Las soluciones son mezclas homogéneas formadas por soluto y solvente.

- Los solutos pueden ser sólidos, líquidos o gaseosos.

- Soluto es lo que vamos a disolver en la solución.

- Al poner en contacto una sal en agua, se pueden obtener distintas soluciones: saturada, sobresaturada o diluida.

- La sal se disocia en $\mathrm{Na}^{+}$, que se une al grupo $\mathrm{OH}^{-}$del agua (Se observa persistencia de concepción alternativa.).

- La solución más concentrada es la que tiene más soluto.

- Lo polar disuelve lo polar.

- Los factores que favorecen la solubilidad son: la temperatura y el calor; cuánto más energía reciba en forma de calor, más se favorece la solubilidad del soluto. (Se observa persistencia de concepción alternativa.)

\section{1) Afirmaciones de valor}

Consideramos que el tema «Soluciones» es de fundamental importancia porque los conceptos son necesarios para otras asignaturas correlativas, para la resolución de problemas en la vida cotidiana y para la práctica de los futuros profesionales. Las características de la carrera hacen que se dé un especial énfasis a los aspectos prácticos, razón por la cual consideramos que debe prevalecer este tema antes que otros como estructura atómica de naturaleza fundamentalmente teórica.

Para el alumno, comprender y asimilar este tema le aportará una buena base para ir incrementando su estructura cognitiva a medida que transcurran sus estudios. Por otro lado, le permite integrar conceptos vistos en otras asignaturas, tales como proporciones, porcentajes y otros del campo de la matemática.

\section{REFLEXIONES FINALES}

Si bien algunas universidades tienen programas que enseñan habilidades de aprendizaje de aplicación muy amplia, que pueden ser útiles en todas o muchas asignaturas, si la enseñanza de los mismos se hace en una clase especialmente dedicada a este fin, se corre el peligro de que los alumnos no relacionen los problemas específicos que realizan en esa clase con el trabajo de cada día en las demás materias escolares. (Se ha podido comprobar que los alumnos cometen menos faltas de ortografía con el profesor de lenguaje que con el profesor de matemáticas, lo cual refleja falta de unanimidad de criterios en la exigencia, por parte de los profesores, y una mentalidad de «estudiar para aprobar» en los alumnos.)

A fin de evitar este problema se puede integrar la enseñanza de estrategias metacognitivas dentro de las asignaturas mismas, para que el alumno pueda apreciar su utilidad en el estudio de las asignaturas. De esta forma se facilitaría el aprendizaje de las estrategias y se consolidarían y automatizarían hábitos permanentes de actuación, como consecuencia del ejercicio diario en el estudio de todas las asignaturas.

Las ventajas obvias de esta estrategia estriba en el hecho de obligar a los alumnos a poner en práctica, dentro del trabajo diario, los principios teóricos del aprendizaje significativo.

Creemos que sería útil, a la luz de estos resultados, lograr que la enseñanza de las distintas asignaturas se impartiese por todos los profesores desde una misma filosofía común y ejerciendo adecuadamente la coordinación entre materias y profesores.

También se puede optar por dedicar, a principios de curso, unas horas de clase a enseñar estrategias en sus líneas generales e integrarlas en las asignaturas, tomando, cada profesor, el rol activo de enseñar a los alumnos a aplicar esos principios generales al estudio de su asignatura concreta. Esto evitaría que todos los profesores tuvieran que explicar y repetir los mismos principios teóricos de base. En resumen, el fin no es sólo que los alumnos conozcan qué estrategias deben usar, sino que además trabajen estratégicamente. Se trata, pues, de crear unas situaciones de aprendizaje que les obliguen a usar las estrategias de forma continuada para que se conviertan en hábito de trabajo y estilo de aprendizaje. Esto no se logra sólo con instrucciones teóricas, sino incorporándolas como guías reales del trabajo escolar diario. A este objetivo primordial fueron dedicados los esfuerzos realizados por los docentes que participamos de este trabajo. 


\section{REFERENCIAS BIBLIOGRÁFICAS}

AUSUBEL, D., NOVAK, J.D. y HANESIAN, H. (1978). Educational Psychology, a cognitive view. (2a. ed.). Nueva York: Holt, Rinehart and Wiston.

AUSUBEL, D., NOVAK, J.D. y HANESIAN, H. (1983). Psicología educativa. Un punto de vista cognoscitivo. (2a. ed.). México: Trillas.

BLOOM, B.S. (1976). Human characteristic and school learning. Nueva York: McGraw Hill.

BURÓN, J. Enseñar a aprender: Introducción a la metacognición. Bilbao: Ediciones Mensajero.

CÁRDENAS, F. A. y GÉLVEZ, C. A. (1998). Química y ambiente (2a. ed.). Nueva York: McGraw Hill.

CHANG, R. (1997). Química. (4a. ed.). McGraw Hill.

CHROBAK, R. (1992). «Learning how to teach Introductory Physics Courses». Tesis de maestría. Cornell University, Ithaca, Nueva York.

CHROBAK, R. (1993). Análisis de las opiniones de los estudiantes sobre la enseñanza de cursos introductorios de física. Actas del Primer Congreso Nacional sobre Problemática de la Enseñanza de la Física en Carreras de Ingeniería, Instancia Final. Paraná, Entre Ríos.

CHROBAK, R. (1995). Uso de estrategias facilitadoras del aprendizaje significativo en los cursos de física introductoria. Revista de Enseñanza de la Física. Asociación de Profesores de Física de la Argentina (APFA), 8 (1), pp. 7-21.

CHROBAK, R. y HERRERA, C. (1996). Experiencia piloto para el desarrollo de un nuevo modelo instruccional. Revista Brasileira de Ensino de Física, 18 (2), pp. 122-136.

FEYMANN, P. (1959). «There's Plenty of Room at the Bottom». Annual meeting of the American Physics Society at California Institute of Technology.

FLAVELL, J.H. (1979). Metacognition and cognition monitoring. American Psychologist, 34, pp. 906-911.
FOUREZ, G. (1997). Alfabetización científica y tecnológica. Buenos Aires: Ediciones Colihue SRL.

GOWIN, D.B. (1981). Educating. Ithaca, Nueva York: Cornell University Press.

JOHNSON MAURITZ, JR. (1967). Definitions and Models in Curriculum Theory. Educational Theory, 17(2), pp. 127-140.

MAGER, R.F. (1962). Preparing Objectives for Programmed Instruction. San Francisco: Fearon.

MINTZES, J.J., WANDERSEE, J.H. y NOVAK, J.D. (1998). Teaching Science for Understanding; a human Constructivist View. San Diego, California: Academic Press.

MOREIRA, M. A. y BUCHWEITZ, B. (1993). Novas estratégias de ensino e aprendizagem Lisboa: Gabinete Técnico de Plátano Editora.

MOREIRA, M.A. y SILVEIRA, F.L. (1993). «Istrumento de Pesquisa em Ensino y Aprendizagem» (edipucrs, Porto Alegre, Brasil), en Mintzes, J.J., Wanderee, J.H. y Novak, J.D. (1998). Teaching Science for Understanding. San Diego, California: Academic Press.

NOVAK, J.D. (1998). Learning, Creating and Using Knowledge: Concept Maps as Facilitative Tools in Schools and Corporations. Mahwah, Nueva Jersey: Lawrence Erlbaum. Trad. cast. (1999). Madrid: Alianza Editorial.

NOVAK, J.D. (1977). A Theory of Education. Ithaca, Nueva York: Cornell University Press. Trad. cast. (1982). Teoría y práctica de la educación. Madrid: Alianza Editorial.

NOVAK, J.D. y GOWIN, D.B. (1986). Learning How to Learn. Nueva York: Cambridge University Press.

NOVAK, J.D. y GOWIN, D.B. (1988). Aprendiendo a aprender. Barcelona: Martínez Roca.

WHITTEN, K.W., DAVIS, R.E. y PECK, M.L. (1999). Química general (5a. ed.) McGraw Hill. 


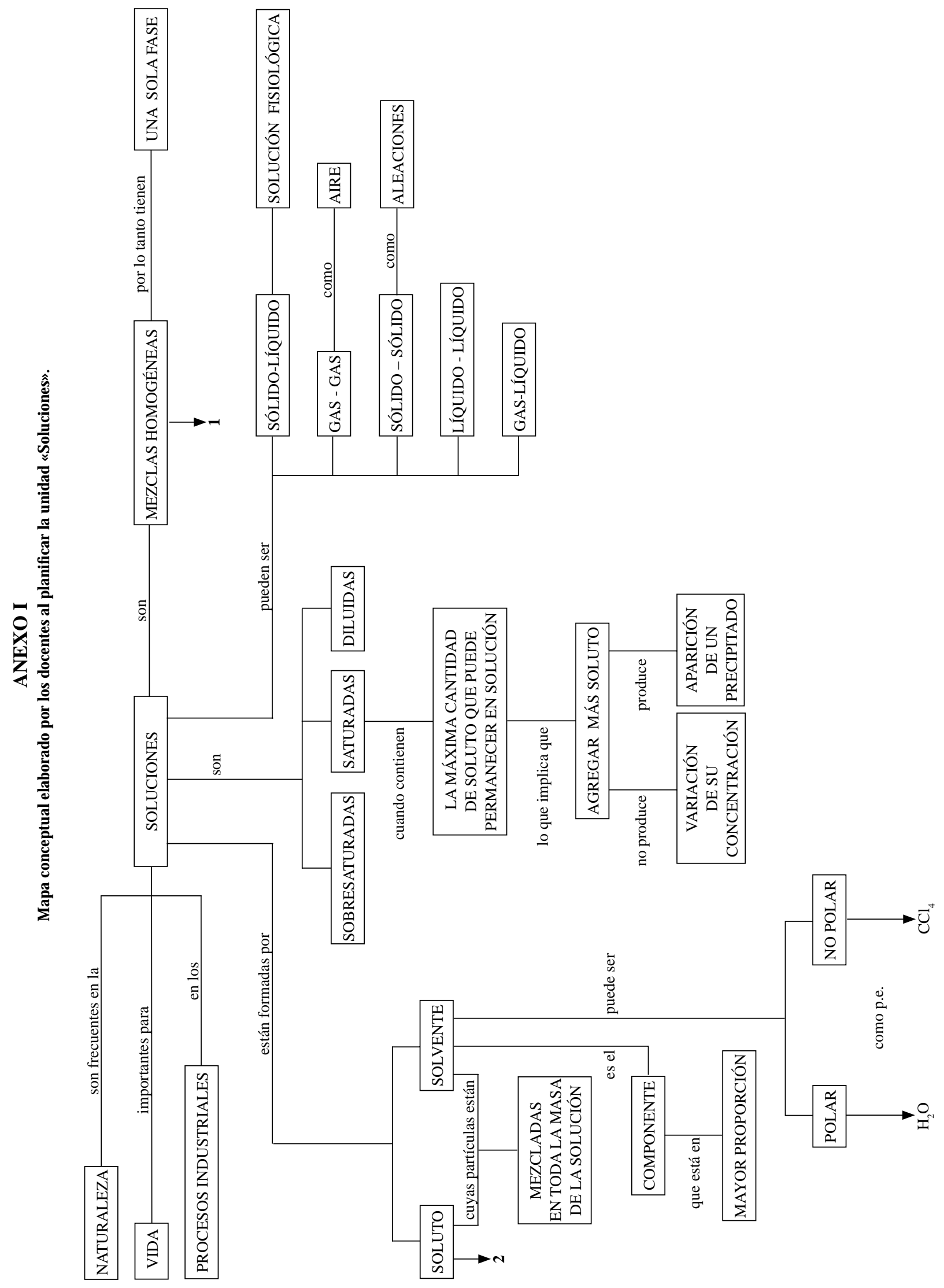


Mapa conceptual 1

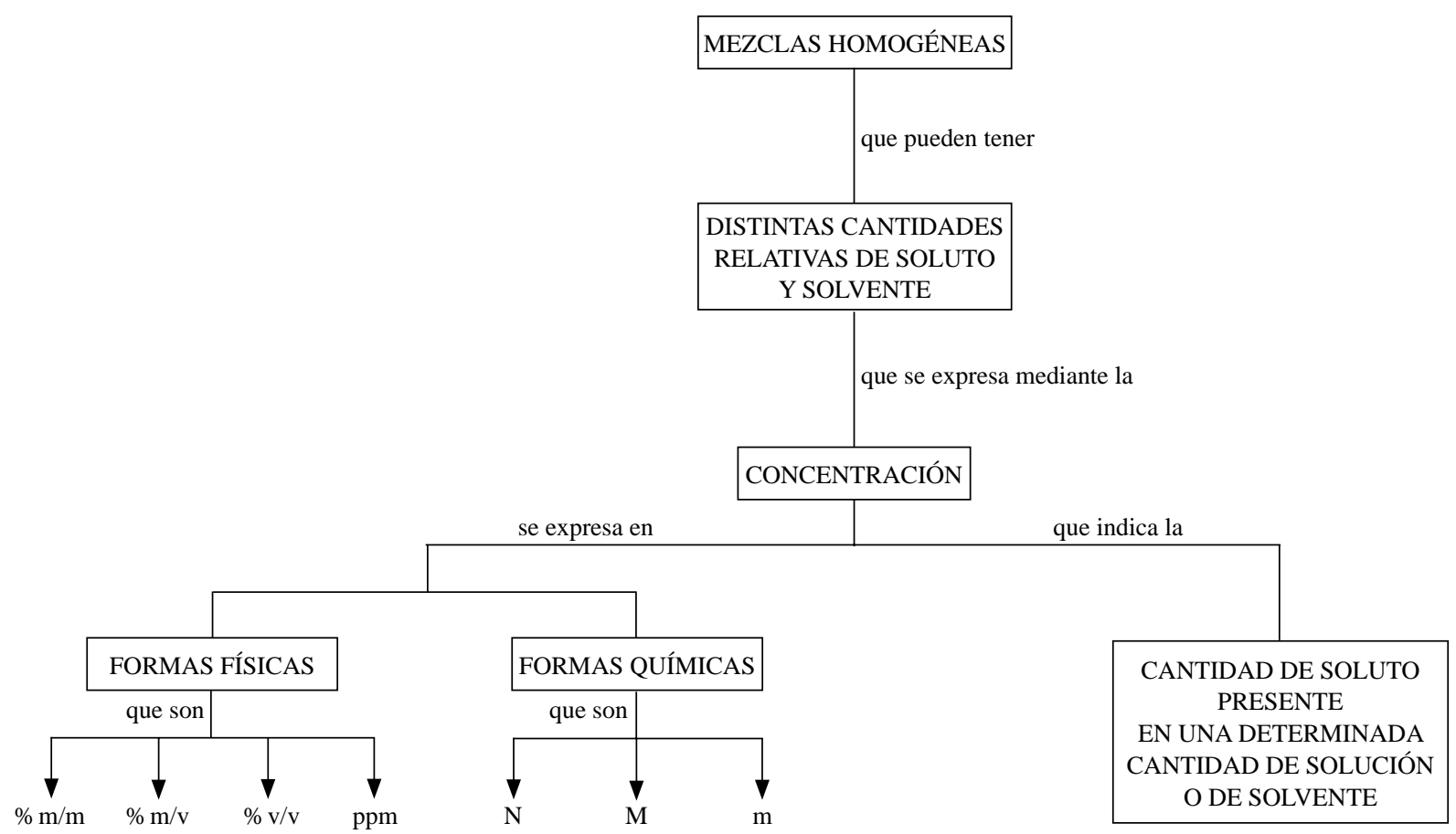




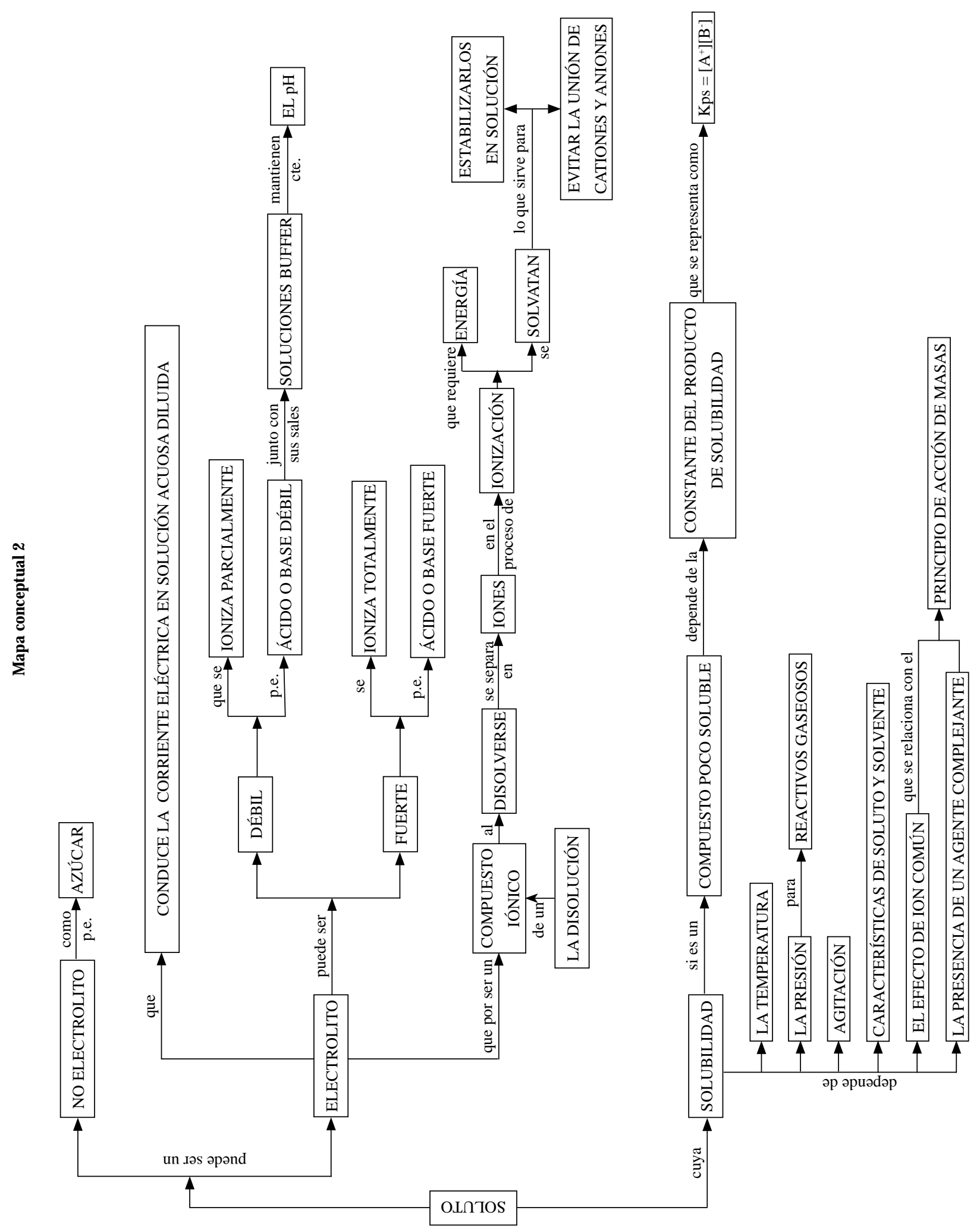




\section{ANEXO II}

E jemplo de mapa conceptual individual realizado por una alumna entrevistada, previo al tratamiento del tema.

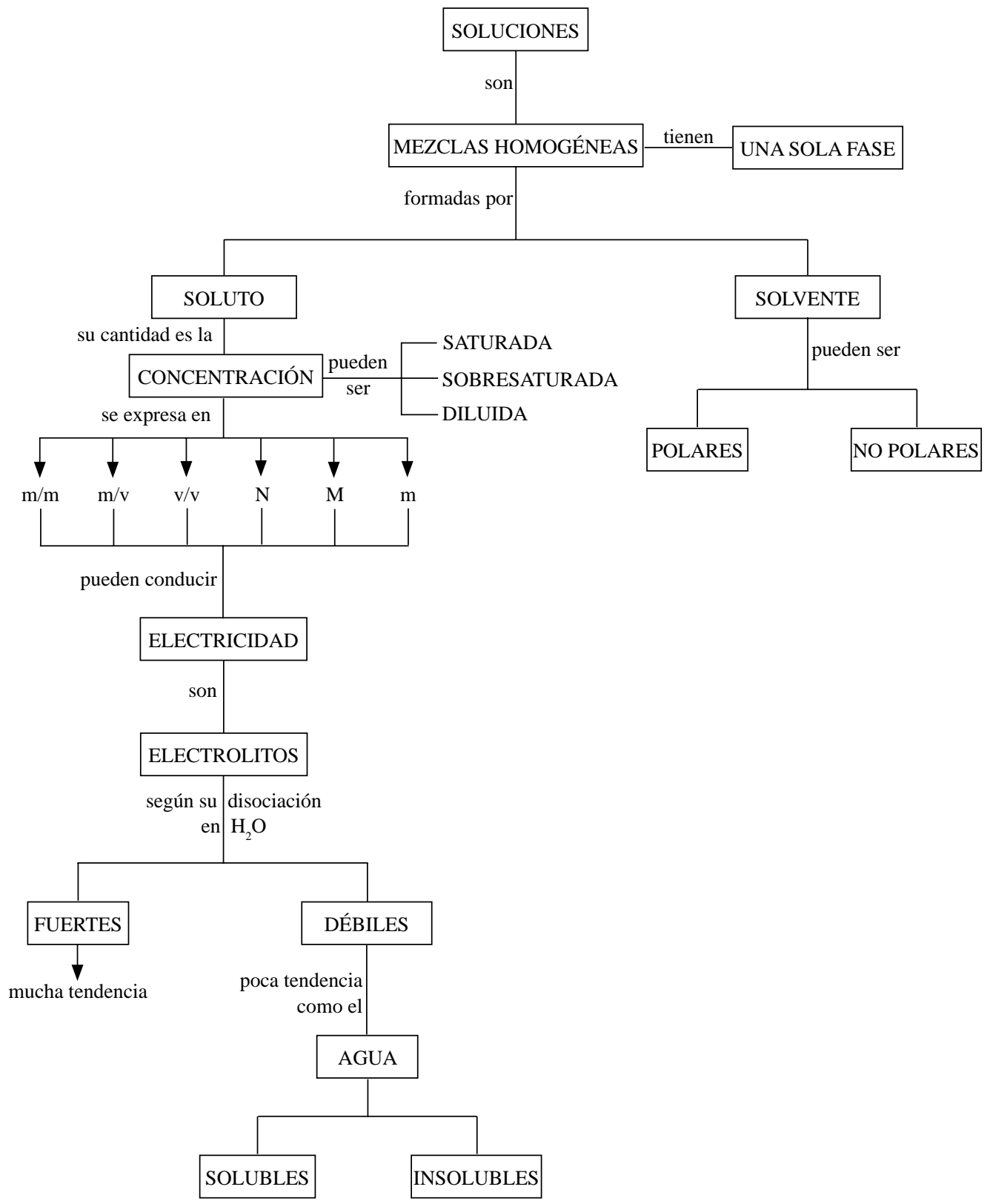




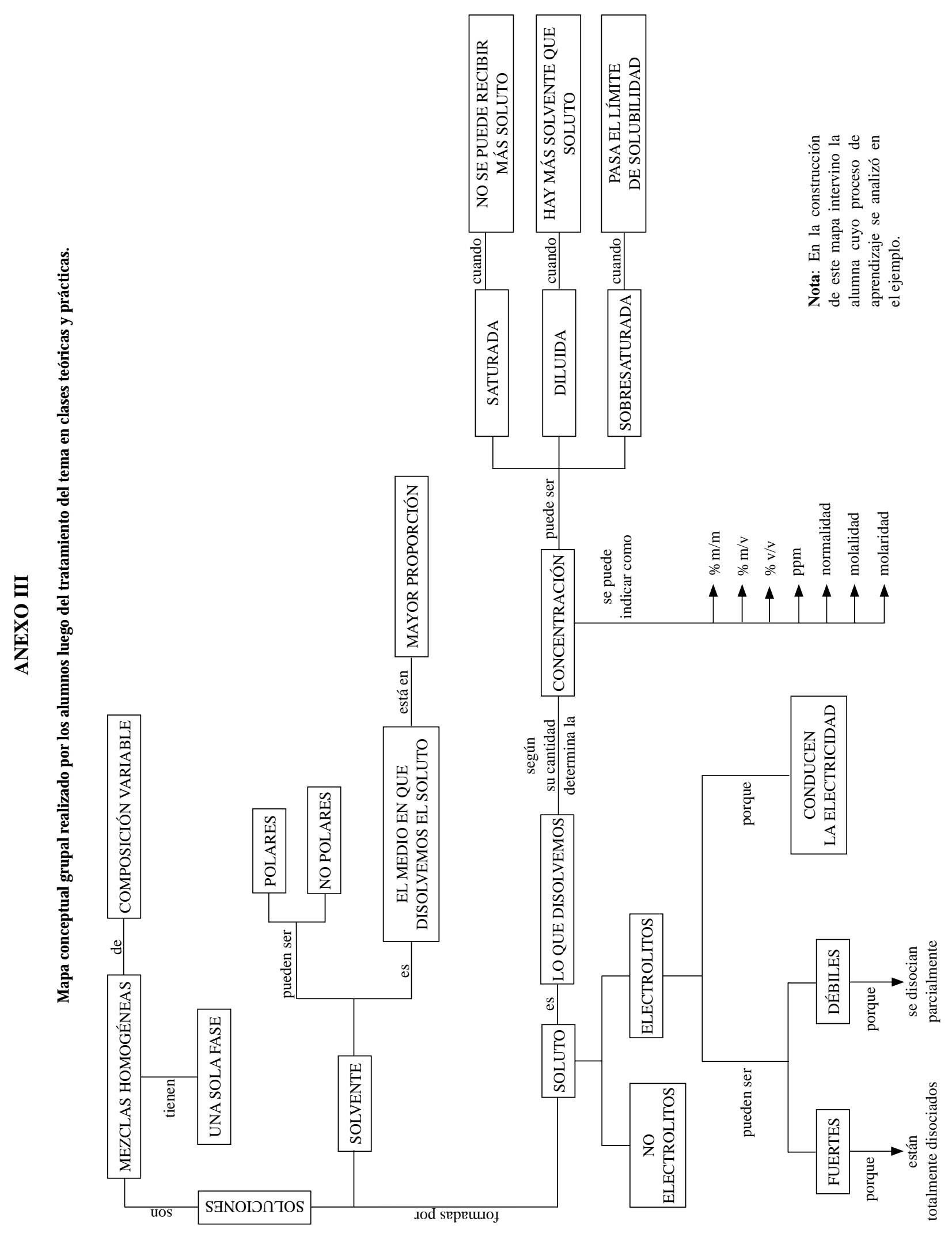




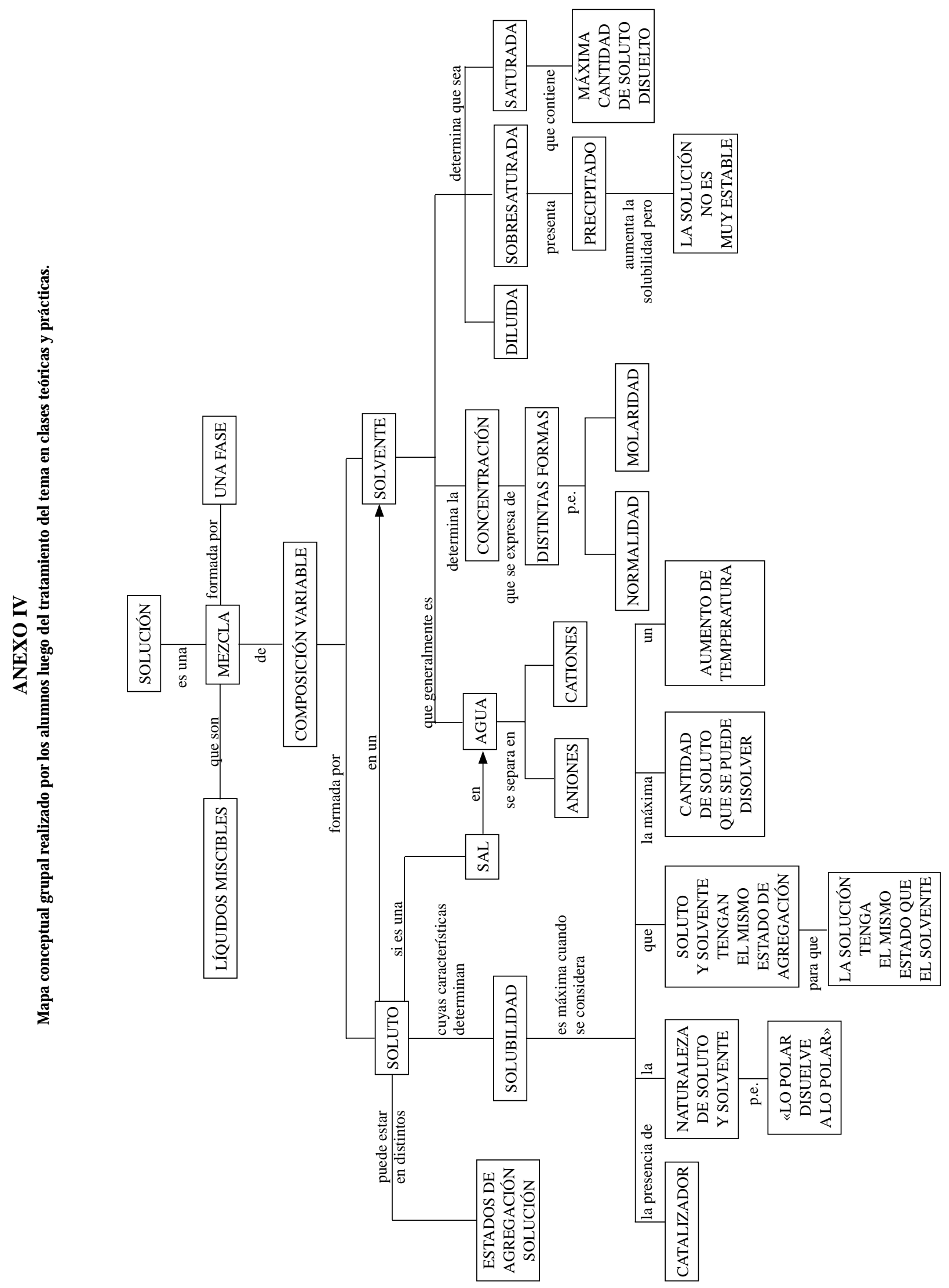

